\title{
SISTEMA DE EXCITACIÓN DE ACCIÓN TRANSVERSAL (TMA) DE UN LASER UV-N2
}

José Antonio Tiburcio Moreno ${ }^{1}$

\begin{abstract}
RESUMEN
El sistema de excitación de acción transversal (TEA) está basado en un proceso de descargas eléctricas de alto voltaje, donde el principal componente lo constituye una fuente de alta tensión (DC), capaz de generar tensiones del orden de los kilovoltios.

Actualmente se está desarrollando un prototipo de este sistema, que permitirá construir dispositivos de radiación láser en el espectro ultravioleta para futuros trabajos de investigación en este campo.
\end{abstract}

\section{INTRODUCCION}

Las fuentes de alta tensión DC son necesarias para el funcionamiento de equipos de radiación láser en el espectro ultravioleta. Durante nuestro trabajo se ha construido una fuente primaria de tensión estabilizada y regulada de 0.0 a 18 voltios de corriente continua; $y$, en segundo lugar, se ha construido una fuente de alta tensión capaz de generar voltajes del orden de los kilovoltios, útil para bombeo de equipos láser de nitrógeno molecular.

Ambas fuentes han sido construidas usando la tecnología de estado sólido (transistores, diodos, etc.). Entre sus principales ventajas se puede mencionar su alto rendimiento y bajo costo en su fabricación, comparado con equipos similares en el mercado nacional.

Destacamos de manera general la teoría y detalles esquemáticos de las fuentes de alimentación de voltaje, asi como el proceso de construcción y funcionamiento de los equipos, materia de estudio.

\section{FUENTES DE ALIMENTACIÓN DE VOLTAJE}

Una fuente de alimentación de voltaje o de poder convierte la corriente alterna ( 220 VCA ) de la línea doméstica en corriente continua ( $C C$ o DC ) para alimentar a diferentes equipos electrónicos de uso

1 Licenciado en física común. Para el análisis o diseño de fuentes de alimentación puede representarse a los equipos electrónicos como una resistencia de carga RL.

\section{DIAGRAMA DE BLOQUES DE UNA FUENTE DE ALIMENTACIÓN}

La fuente de alimentación consta de las siguientes partes:

Transformador de entrada: Reduce el voltaje de la línea o red CA al voltaje de CA, necesario para lograr un voltaje de salida CC o DC adecuado.

Circuito rectificador:Está conformado por uno o más diodos de silicio. Su misión es dejar pasar los semiciclos positivos o negativos de la tensión de CA entregada por el transformador de entrada. A este proceso se conoce como de rectificación de la onda positiva o negativa, según sea el caso.

Filtraje: Constituido generalmente por condensadores mayores de $100 \mathrm{~F}$ con o sin bobinas de choque. Se encarga de darle "pureza" o "aplanar" la tensión rectificada.

Estabilizador de Voltaje: Mantiene constante el voltaje de salida de la fuente entre variaciones de consumo de corriente del equipo o carga conectada en los terminales de salida.

Regulador de Voltaje Variable:Compuesto básicamente por una resistencia variable de tipo lineal y semiconductores asociados. Ajustar el voltaje de salida CC de la fuente al voltaje de la carga o equipo conectado en los terminales de salida de la fuente. 
dispositivos y componentes electrónicos se deterioren por el efecto del cortocircuito oexcesivo consumo de corriente en la salida de la fuente.

\section{CLASES DE FUENTES DE ALIMENTACIÓN}

I. Por el tipo de rectificador

1.Rectificador de media onda.

2.Rectificador de onda completa:

- Con derivación central.

- Tipo puente.

II.Por la estabilización de voltaje de salida

1.Fuentes de alimentación no reguladas

2.Fuentes de alimentación reguladas y fijas.

3.Fuentes de alimentación reguladas y vari ables

\section{RECTIFICADOR DE MEDIA ONDA}

Es el tipo de rectificador más simple, su forma circuital se muestra en la siguiente figura:

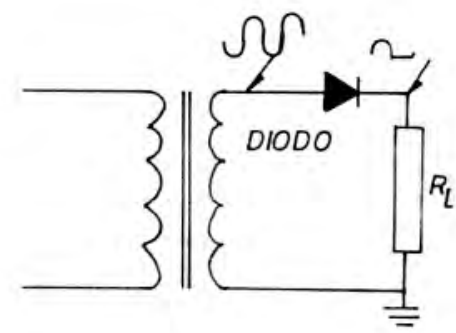

Fig. 1 Rectificador de media onda con voltaje de salida positivo.

El diodo rectificador conduce cuando el ánodo es positivo, es decir, durante los semiciclos positivos. Cuando el ánodo del diodo es negativo (semiciclos negativos de la corriente alterna) se polariza de modo inverso y no circulará ninguna corrienteeléctrica en el circuito.

Conectando el diodo al revés, se obtiene en la salida de la fuente una corriente pulsante negativa y el sentido de circulación de la corriente es inverso.

Cuando a un circuito rectificador se conecta en los bordes de salida un condensador de filtro, pasa a llamarse fuente de alimentación.

El condensador de filtro presenta la característica de "llenar" los vacíos entre semiciclos y convierte la corriente pulsante en corriente continua casi pura.

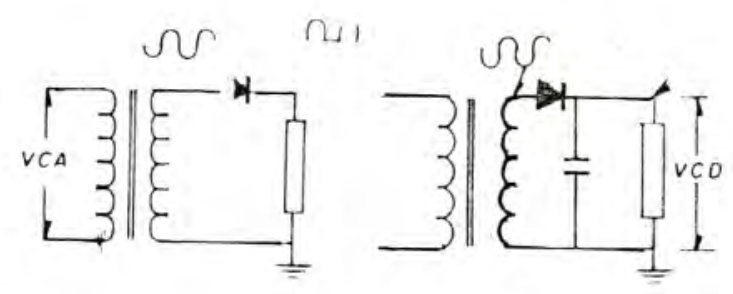

Fig. 2 Rectificadores: (a) sin filtro, (b) con filtro

\section{RECTIFICADOR DE ONDA COMPLETA CON DERIVACION CENTRAL}

El circuito se reconoce porque el devanado secundario del transformador de entrada tiene una toma intermedia y los diodos conectados en cada extremo del secundario del transformador y unidos por el cátodo.

La carga se conecta entre la unión común de los diodos y la derivación central del secundario (punto de tierra).

Debido a la derivación central, los voltajes en los extremos del secundario, con relación al punto medio, son opuestos, haciendo que conduzca sólo un diodo en un momento determinado. Cada diodo se comporta como un rectificador de media onda, pero por tener en común la carga de salida, viene a ser la suma de ambas corrientec narciales.

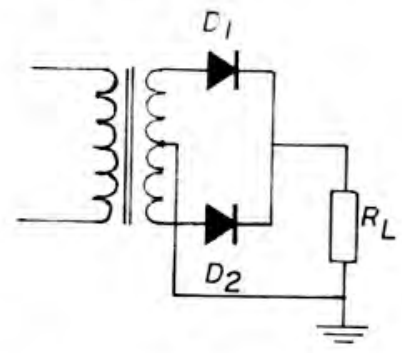

Fig. 3 Rectificador de onda completa con derivación central
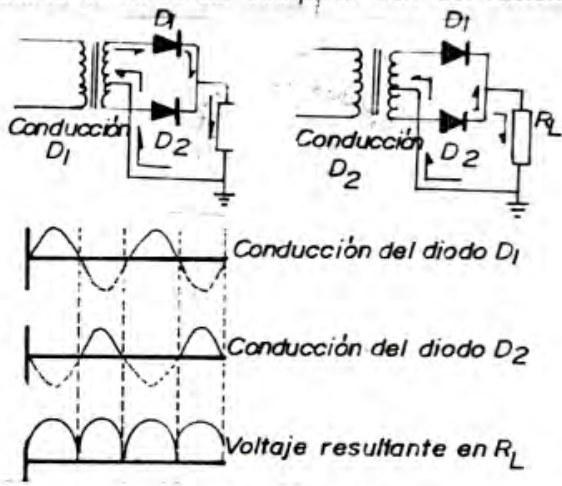

Fig. 4 Conducción del rectificador de onda completa con derivación central. 
De modo similar, cuando a este circuito se le conecta un condensador de filtro a la salida de los diodos, pasa a denominarse fuente de alimentación de onda completa con derivación central.

\section{RECTIFICADOR DE ONDA COMPLETA TIPO PUENTE}

A diferencia del circuito anterior, éste posee cuatro diodos y el devanado secundario del transformador de entrada, no tiene derivación central. En este caso, cada par de diodos y el devanado secundario, actúan como rectificadores de media onda
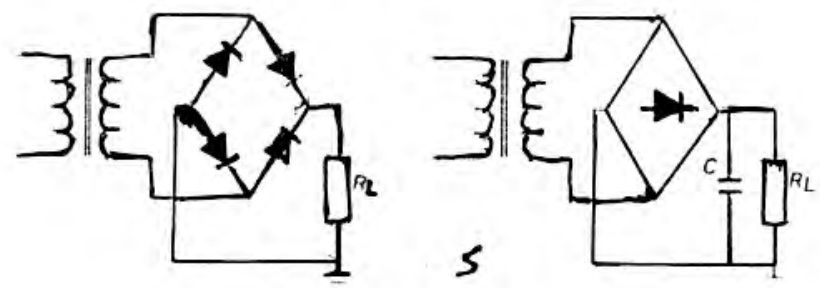

Fig. 5 :(a) Rectificador de onda completa tipo puente.

(b) Fuente de alimentación de onda completatipopuente.

$\mathrm{Al}$ incorporarse un condensador de filtro, pasa allamarse fuente de alimentación de onda completa tipo puente.

Fuentes de alimentación no reguladas: $\mathrm{Su}$ voltaje de salida es inestable, porque varía de acuerdo con el consumo de la carga $R L$ o el equipo conectado en los terminales de salida de la fuente.

Fuentes de alimentación reguladas y fijas:Entregan un voltaje de salida DC estable, independiente de la carga RL conectada en losterminales de salida de la fuente.

Fuente de alimentación reguladas y variables: Presentan la particularidad de poder seleccionar el voltaje de salida DC desde un mínimo, generalmente de 00 voltios hasta un voltaje deseado (12,18 voltios, etc.).

Fuentes de alimentación reguladas y fijas con circuito integrado: Un circuito integrado ( $\mathrm{Cl} \mathrm{O} \mathrm{IC}$ ) es un dispositivo electrónico en donde las resistenciasdiodos, transistores, etc. se encuentran interconectados en una cápsula llamada "chip".

La mayoria de $\mathrm{Cl}$, reguladores devoltaje, poseen tres terminales. Entregan voltajes de salida fijos que sonutilizados en un amplio rango de aplicaciones talecomo: sistemas de cómputo, equipos de instrumentación, de alta fidelidad, y en muchos otros equipos electrónicos. Sin embargo, estos "chips",con el agregado de componentes externos, pueden ser utilizados para obtener voltajes y corrientes variables.
Como parte integral del proyecto se ha construido, en una primera etapa, una fuente de voltaje de corriente continua (para alimentar a la fuente de alta tensión) que presenta un rectificador de onda completa tipo puente y un circuito de protección para no deteriorar los demás componentes. Incluye un circuito estabilizador para entregar un voltaje variable en un rango de 00 a 18 VDC. El diagrama esquemático y los componentes utilizados se presentan en la figura 10.

El circuito ha sido ensamblado en una caja metálica de las siguientes dimensiones: $26 \mathrm{~cm}$ de largo, $15 \mathrm{~cm}$ de ancho y $12 \mathrm{~cm}$ de altura.

Posee además, terminales identificados para entrada de VCA y de salida VDC. También se puede apreciar un interruptor ON/OFFy un indicador LED de color rojo que marca el funcionamiento. También presenta una escala zon indicador de aguja conectada a un potenciómetro que determina el voltaje de salida deseado.

\section{FUENTE DE VOLTAJE DE ALTA TENSIÓN CONTI- NUADA}

Esta fuente está basada en la etapa de generación horizontal de un equipo receptor de televisión convencional, donde la alta tensión se obtiene de una fuente de tipo especial denominada "kick" o "flyback". De este tipo de fuente deriva la alta tensión, aprovechando la tensión inductiva de retroceso, desarrollada en el transformador de salida del amplificador de barrido horizontal, por la porción del retrasado de la onda diente de sierra. Para tener una mejor idea del funcionamiento de esta fuente, vamos a describir a continuación algunos componentes fundamentales:

Osciladores de diente de sierra:La mayoría de los osciladores de diente de sierra (o generadores de barrido) basan su funcionamiento en la carga y descarga de un condensador. Cuando un condensador y una resistencia se conectan en serie, a través de una fuente de tensión continua, la tensión a través del condensador varía con el tiempo, observándose que el ciclo de carga o de aumento de la tensión no es instantáneo ni lineal, sino que partiendo de cero, alcanza un valor máximo con la forma de variación característica de una curva de tipo exponencial. EL tiempo que el condensador toma para aproximarse al valor máximo de tensión, depende de la constante de tiempo que se define de la siguiente manera: $=\mathrm{RC}$.

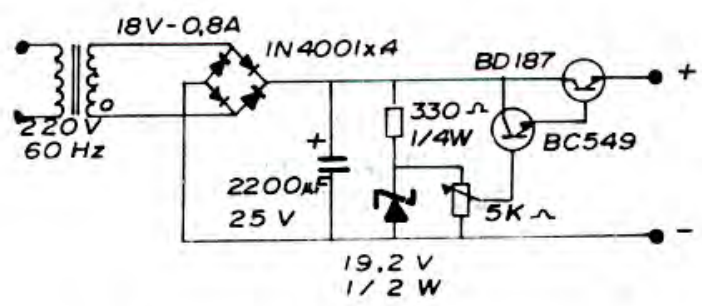

Fig. 6 :Fuente de alimentación estabilizada y variable de $0-18$ VDC. 

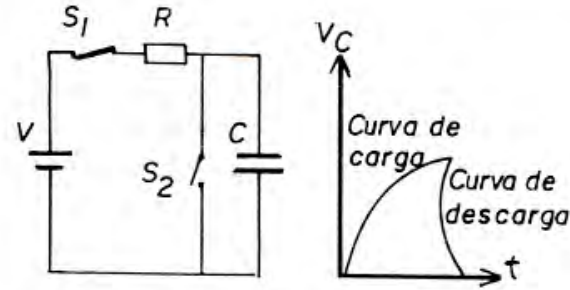

Fig. 7 :Carga y descarga de un condensador.

En la figura anterior, cuando se abre S1 y se cierra S2, el condensador se descarga en mucho menos tiempo que el que se empleó en cargarlo, a causa de que no hay, ahora, una resistencia de valor grande que limite la corriente. Se nota de la primera parte de la curva de carga que es bastante lineal y que por lo tanto, podría aprovechársele para formar la porción de trazado de la onda diente de sierra.

El oscilador de diente de sierra es un circuito que permite que el condensador se cargue durante sólo la porción linea! de su curva de carga y que provoque después la descarga brusca del mismo. El ciclo se repite indefinidamente para producir ondas de diente de sierra mantenidas y de la frecuencia necesaria.

Evidentemente, no es práctico usar interruptores con este fin, por ello se recurre a la aplicación de la conmutación electrónica por medio de transistores.

Circuitos de barrido horizontal: Estos circuitos, además de suministrar corriente suficiente y de forma de onda adecuada a las bobinas deflectoras, desempeñan funciones adicionales de suministrar alta tensión, amortiguar los transistores durante el retrasado y recuperar parte de la energía magnética para aumentar la tensión anódica de la etapa amplificadora.

El gererador horizontal es un oscilador de autobloqueo donde la tensión de diente de sierra, que se desarrolla a través de los condensadores de descarga, se acopla a los terminales de salida y permite a la corriente de diente de sierra circular a través de las bobinas deflectoras, pero bloqueando a la corriente continua.

Incluye además, un diodo amortiguador, que es un rectificador, utilizado primordialmente para amortiguar las oscilaciones transitorias, creadas por la rápida dismin ıción de la corriente a través de las bobinas, durante el retrasado, y a la vez, retener el pulso de tensión positiva en el primario del transformador, pulso que se aprovecha en la fuente de altatensión. También sirve para contribuir a la obtención de un trazado lineal y recuperar parte de la energía magnética.

Fuente de alta tensión por "flyback": Además de utilizarse una parte de la energía magnética para aumentar el rendimiento de la etapa amplificadora de barrido, algo de la misma energía se aprovecha para desarrollar la alta tensión necesaria.

Durante el período de retrasado, cuando el campo magnético empieza a decaer, se desarrolla un pulso de tensión de unos 1000 voltios a través de la bobina deflectora. Este pulso de tensión aparece como un pulso de mucha mayor amplitud a través del primario del transformador. Este golpe inductivo alcanza en el primario unos 4000 voltios y es aumentado aún más por medio de un arrollamiento adicional, conectado en serie con el primario. El arrollamiento adicional, por efecto del autotransformador, eleva la tensión a algo más del doble y un pulso de unos 9500 voltios aparece en la placa del rectificador de media onda de alta tensión.

A causa de la frecuencia relativamente alta de los pulsos, que es de $15625 \mathrm{~Hz}$, se necesita muy poco filtro y un condensador de $500 \mathrm{~F}$ es más que suficiente para este propósito. Además, de que en un condensador de baja capacidad no puede almacenarse una carga peligrosa y la fuente resulta así relativamente segura.

En la segunda etapa del proyecto, se ha considerado la construcción de la fuente de alta tensión, cuyo primario de funcionamiento se ha descrito anteriormente. El diseño adecuado para nuestro propósito ha sido evaluado convenientemente con resultados satisfactorios. El esquema analítico del circuito se muestra en la figura 13.

En la construcción de la fuente de alta tensión se han utilizado los siguientes materiales:

Resistencias:

$R 1=680 \pm 2 \%$
$R 2=8,2 K \pm 1 \%$
$R 3=1,8 K \pm 5 \%$
$R 4=27 \pm 1 \%$

$R 5=39 K \pm 5 \%$

$R 6=560 \pm 2 \%$

$R 7=56 \pm 5 \%$

$\mathrm{R} 4=27 \pm 1 \%$

Condensadores:
$\mathrm{C} 1=3,3 \mathrm{pF} / 50 \mathrm{~V}$
$\mathrm{C} 2=140 \mathrm{~F}$
$\mathrm{C} 3=0,0022 \mathrm{~F}$
$\mathrm{C} 4=0,047 \mathrm{pF} / 100 \mathrm{~V}$
$\mathrm{C} 5=823 \mathrm{~F} / 50 \mathrm{~V}$
$\mathrm{C} 6=103 \mathrm{M}$

$\mathrm{R} 8=18 \pm 1 \%$

Diodos:

$\mathrm{D} 1=\mathrm{RGP} 15 \mathrm{D}$
$\mathrm{D} 2=1510 \mathrm{G}$

Transistores:

$\mathrm{T} 1=\mathrm{C} 380$

$\mathrm{T} 2=\mathrm{C} 536$

$\mathrm{T} 3$ = 2 SC 1192

Otros Componentes:

$\mathrm{L} \quad=$ Bobina tipo TDI $1213 \mathrm{KH}$

TLN = Transformador tipo 826 
$\mathrm{FB}=$ Flyback de TV B/N 12" SAMSUNG

Estos componentes de circuito fueron ensamblados en una caja de madera con las dimensiones antes citadas.

El equipo presenta conectores tipo "plush", tanto para la entrada como para la salida, debiendo tener cuidado con la polaridad. Además, el equipo construido tiene incorporado un interruptor ON/OFF con indicador LED para observar el funcionamiento

Estafuente dealtatensión sealimenta mediante un voltaje DC variable, proveniente de la fuente primaria y contiene una etapa polarizadora y un transformador "drive" para fijar una frecuencia de oscilación de aproximadamente $15750 \mathrm{~Hz}$, quellega hasta el "flyback" para generar alta tensión por los procesos descritos.

\section{alta tensión:}

Características técnicas de la fuente de

La fuente de alta tensión es un equipo que está diseñado para que proporcione una tensión de salida en el rango de $2 \mathrm{KV}$ hasta $9,5 \mathrm{KV}$ en el modo DC. Además, puede proporcionar una corriente de hasta 1 $\mathrm{mA}$ hacia la carga, que en nuestro caso sería un sistema láser de nitrógeno molecular.

La variación de la tensión de salida está en función de la tensión de alimentación de corriente continua de la fuente primaria. Este voltaje está en el rango de 00 a 18 VDC

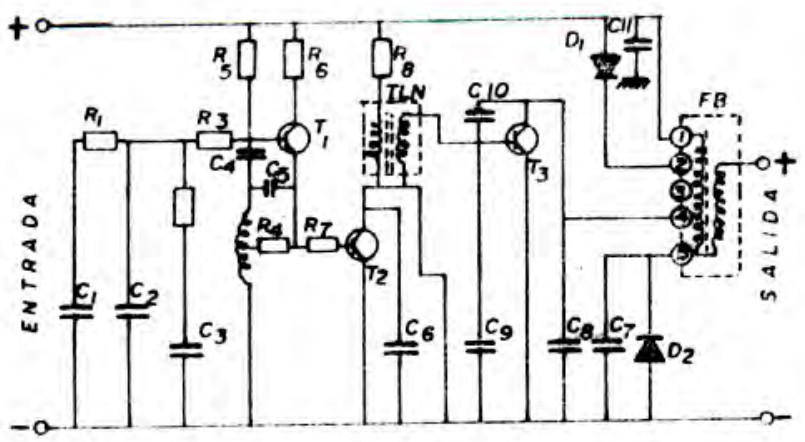

Fig. 8 :Diagrama esquemático de la fuente de alta tensión.

\section{REFERENCIAS BIBLIOGRÁFICAS}

\section{García, J. 1993, Fuentes de alimentación reguladas y} variables. CIEIN, Lima.

Malasquez, V.; Morales, O. 1987, Diseño y Construcción de una fuente de alta tensión DC, para un equíjo de rayos láser_superradiante (N2) puizsnte. Universidad Nacional de Ingeniería, Lima.

\section{CONCLUSIONES}

1. Por tener características de funcionamientos diferentes, la fuente de alta tensión debe construirse siempre separada de la fuente primaria de alimentación, para no lamentar pérdidas inútiles de componentes.

2. La fuente de alimentación DC debe tener incluido un circuito estabilizador de voltaje con diodo Zener, para no permitir variaciones en el voltaje entregado y posibilitar un mejor funcionamiento de la fuente de alta tensión.

3. La fuente de alta tensión de salida, cuando se le conecta una carga (en el caso de bombeo de un láser de gas) tiene un comportamiento pulsante, porque el láser consume una alta energía instantánea, lo que motiva que la alta tensión de salida descienda bruscamente para luego recuperarse y repetir la secuencia.

4. La fuente de alta tensión puede ser usada, tanto para el sistema láser trabajando en condiciones atmosféricas con el nitrógeno del aire, como para un láser con sistema de vacío, aumentando en este caso su potencia.

\section{INDICACIONES PARA INVESTIGACIONES POSTERIORES}

Para aumentar el voltaje de salida de la fuente de alta tensión debe ambiarse el "flyback"del circuito de ₹ Fig. 13, por uno correspondiente a un receptor de TV at 14 "B/N

Tiburcio, A. 1991, Diseño y Construcción de un láser de nitrógeno molecular. Tesis, Universidad Nacional de Trujillo.

Aldama, W. 1985, Desarrollo de la Espectroscopía láser para aplicaciones metalúrgicas, Univ. Nac. de Trujllo.

Valera, A. 1981, Diseño y Construcción de un sistema láser de colorante. Rev. Peruana de Física, Vol. 1, Lima. 